
JNM
J Neurogastroenterol Motil, Vol. 22 No. 4 October, 2016
pISSN: 2093-0879 elSSN: 2093-0887
http://dx.doi.org/10.5056/jnm16007

\title{
Repeated Water Avoidance Stress Alters Mucosal Mast Cell Counts, Interleukin-1 $\beta$ Levels with Sex Differences in the Distal Colon of Wistar Rats
}

\author{
Ju Yup Lee, ${ }^{1,3}$ Nayoung Kim, ${ }^{1 *}$ Yong Sung Kim, ${ }^{4}$ Ryoung Hee Nam, ${ }^{1}$ Min Hee Ham, ${ }^{1}$ Hye Seung Lee, ${ }^{2}$ Wonjun Jo, \\ Youngkwang Shim, ${ }^{1}$ Yoon Jin Choi, ${ }^{1}$ Hyuk Yoon, ${ }^{1}$ Cheol Min Shin, ${ }^{1}$ and Dong Ho Lee ${ }^{1}$ \\ Departments of ${ }^{1}$ Internal Medicine and ${ }^{2}$ Pathology, Seoul National University Bundang Hospital, Seongnam, Gyeonggi-do, Korea; ${ }^{3}$ Department \\ of Internal Medicine, Keimyung University School of Medicine, Daegu, Korea; ${ }^{4}$ Department of Gastroenterology and Digestive Disease Research \\ Institute, Wonkwang University School of Medicine, Iksan, Jeollabuk-do, Korea; and ${ }^{5}$ Department of BioNano Technology and Gachon BioNano \\ Research Institute, Gachon University, Seongnam, Gyeonggi-do, Korea
}

\section{Background/Aims}

This study was aimed at evaluating differences in the effects of repeated water avoidance stress (rWAS) on colonic movement, mucosal mast cell counts, cytokine levels, and visceromotor response (VMR) to colorectal distension (CRD) in rats of both sexes.

\section{Methods}

Wistar rats were divided into stress and no-stress groups. Rats in the stress group were exposed to rWAS (1 hr/day) for 10 days. Mucosal mast cells were immunohistochemically stained with anti-mast cell tryptase antibody and counted. The colonic mucosal cytokine levels were measured with enzyme-linked immunosorbent assay. The VMR to CRD (visceral analgesia) was assessed by using a barostat and noninvasive manometry.

\section{Results}

The mean number of fecal pellets in the rWAS group increased significantly as compared with that in the no-stress group in both sexes. After adjustment for body weight, the female rats had a significantly higher pellet output than the male rats. The mucosal mast cell count of the female rWAS group was higher than that of the male rWAS group $(13.0 \pm 0.9$ vs $8.8 \pm 0.6 ; P<0.001)$. The colonic mucosal interleukin-1 $\beta$ level was also higher only in the female rats of the rWAS group than in those of the no-stress group. On days 10 and 11, a decrease in VMR to CRD was observed at 40 and $60 \mathrm{mmHg}$ in both sexes of the rWAS group, without a sex-based difference.

\section{Conclusions}

The colonic response to stress appeared to be more sensitive in the female rats than in the male rats. However, stress-induced visceral analgesia had no sex-related difference and the underlying mechanism needs to be further evaluated.

(J Neurogastroenterol Motil 2016;22:694-704)

Key Words

Cytokines; Mast cells; Water avoidance stress

Received: January 10, 2016 Revised: May 23, 2016 Accepted: June 14, 2016

(a) This is an Open Access article distributed under the terms of the Creative Commons Attribution Non-Commercial License (http://creativecommons. org/licenses/by-nc/4.0) which permits unrestricted non-commercial use, distribution, and reproduction in any medium, provided the original work is properly cited.

${ }^{*}$ Correspondence: Nayoung Kim, MD, PhD

Department of Internal Medicine, Seoul National University Bundang Hospital, 82, Gumi-ro 173 beon-gil, Bundang-gu, Seongnam, Gyeonggi-do 13620, Korea

Tel: +82-31-787-7008, Fax: +82-31-787-4051, E-mail: nayoungkim49@empas.com 


\section{Introduction}

Irritable bowel syndrome (IBS) is a common functional bowel disorder of unclear etiology that is characterized by recurrent abdominal pain and altered bowel habits without structural abnormalities. ${ }^{1}$ The possible pathogeneses of IBS includes stress, increased mucosal permeability, mucosal immune activation, food hypersensitivity, transient infection altering intestinal microbiota, neuroplasticity, altered enteroendocrine metabolism, and genetic polymorphisms. ${ }^{2,3}$ Among these, stress is a key factor in the onset, maintenance, and aggravation of IBS symptoms. ${ }^{4}$ Stress induces changes of colonic functions, including increase of permeability, mucus secretion, motility, myenteric nerve activation, serotonin release, and the development of visceral hypersensitivity in rats. ${ }^{5-7}$ In particular, water avoidance stress (WAS) is a well-known animal model of stress that increases the psychological component and imitates the experience of ongoing environmental life in humans. ${ }^{8}$ It has been revealed that WAS increased fecal pellet output (FPO), colonic mast cell count, mucosal cytokine levels, and intestinal permeability. In view of visceral pain responses, WAS has been reported to induce different responses (hyperalgesia vs analgesia) in a differently conditioned rodent model. ${ }^{10}$ That is, mice that had undergone surgery for electromyographic electrode implantation and were single-housed subsequently to avoid deterioration of the electrodes by cage mates, developed visceral hyperalgesia to colorectal distension (CRD) in response to repeated WAS (rWAS). By contrast, mice that were tested by using the noninvasive manometric intracolic pressure (ICP) measurement catheter without surgery and kept group-housed developed visceral analgesia under the same rWAS conditions. ${ }^{11}$

The importance of mast cells in IBS pathophysiology has been shown by many studies. ${ }^{12}$ Numerous numbers of mast cells have been identified in the gastrointestinal tract of patients with IBS. ${ }^{13}$ In the colon, mast cells close to nerves are markedly correlated with the severity and frequency of abdominal pain and discomfort in patients with IBS. ${ }^{14}$ Hyperplasia and activation of mast cells, in addition to the infiltration of neutrophils and mononuclear cells, and increased myeloperoxidase activity, were observed in the mucosa, and mast cells induced ultrastructural changes in the epithelial cells in rat intestine. ${ }^{15}$ The expressions of pro-inflammatory cytokines such as interleukin (IL)- $1 \beta$ and interferon (IFN)- $\gamma$ also increased after chronic WAS. ${ }^{8}$ These changes in cytokine levels are suggestive of low-grade immune activation, another possible mechanism in some parts of the patients with IBS. . $^{14,16-18}$
According to a previous meta-analysis, IBS is more prevalent in women, with a female-to-male sex odds ratio of 1.67 (95\% confidence interval, 1.53-1.82). ${ }^{19}$ Women are more vulnerable to stress, are more prone to develop anxiety and depression, are exposed to trauma more often than men are, and present with IBS symptom exasperation when under stress. Therefore, sex-specific differences in stress responses could explain the female predominance of IBS. ${ }^{20}$ So far, several studies have focused on sex-specific stress response and visceral hypersensitivity. ${ }^{21-26}$ To our knowledge, the sex-related differences between all kinds of parameters have not been fully described in a single report. Therefore, we aimed to elucidate differences in colonic movement, mucosal mast cell counts, cytokine levels, and visceral sensitivities between the 2 sexes in a stress rat model.

\section{Materials and Methods}

\section{Animals}

Male and female Wistar rats (Orient Co, Ltd, Seoul, Korea) were housed in a cage (2/cage) kept at a temperature of $23^{\circ} \mathrm{C}$, with a 12-hour of light/dark cycle. Moreover, they were housed under specific pathogen-free conditions, with ad libitum-only Purina rat chow and water, without enrichment. After 1 week of adaptation, 7-weekold male and female Wistar rats weighing 216-282 and 158-202 g, respectively, were used. All of the experimental procedures were approved by the Institutional Animal Care and Use Committee (IACUC) of Seoul National University Bundang Hospital (IACUC No. BA1403-149/016-01).

\section{Repeated Water Avoidance Stress}

The rWAS was delivered as described elsewhere. ${ }^{27}$ To avoid the stress response variation related to a more comfortable position on the platform of female rats that have smaller body weight than male rats, we used different sizes platforms for male and female rats. That is, depending on sex, each Wistar rat was placed on a different glass pedestal $(5.8-\mathrm{cm}$ length $\times 5.8-\mathrm{cm}$ width $\times 6.0$ - $\mathrm{cm}$ height for male rats and $5.0-\mathrm{cm}$ length $\times 5.0-\mathrm{cm}$ width $\times 6.0-\mathrm{cm}$ height for female rats), which had been fixed to the center of a standard plastic cage (26.7-cm length $\times 48.3-\mathrm{cm}$ width $\times 20.3-\mathrm{cm}$ height). The plastic cage was filled with $25^{\circ} \mathrm{C}$ water to $1 \mathrm{~cm}$ below the height of the platform for 1 hour for 10 consecutive days between 8 and 10 AM. Rats in the sham stress group were placed on the same platform without water for 1 hour. All rats were housed in pairs in their home cage and placed individually in their WAS cage, and fecal 
pellet output was monitored during the WAS session.

\section{Measurement of Fecal Pellet Output}

The total number of fecal pellets expelled in each cage was counted at the end of each 1-hour session. Accounting weight differences between the male and female rats, the result was expressed per $100 \mathrm{~g}$ of body weight.

\section{Assessment of Visceral Pain Response to Colorectal Distension}

This visceral pain response to CRD was evaluated by using the noninvasive manometric method that Larauche et $\mathrm{al}^{27}$ recently developed and validated. Briefly, a PE 50 catheter was taped 3.5 $\mathrm{cm}$ below the pressure sensor of a pressure transducer catheter (SPR-524 Mikro-Tip catheter; Millar Instruments, Houston, TX, USA). A custom-made balloon $(2 \mathrm{~cm}$ wide $\times 5 \mathrm{~cm}$ long) made of polyethylene plastic bag was tied over the catheter at $1 \mathrm{~cm}$ below the pressure sensor with a silk suture. Isoflurane $\left(3 \%\right.$ in $\left.\mathrm{O}_{2}\right)$ was used for anesthesia, and the catheter with lubricated balloon-pressure sensor was inserted into the rectum and distal colon. The distal end of the balloon was positioned $1 \mathrm{~cm}$ from the anus and the catheter was fixed to the tail with tape. Each rat was left in a Bollman cage covered with a blanket and rested for 30 minutes before the CRD procedure. Each balloon was connected to the barostat (Distender Series IIR Dual Drive Barostat; G \& J Electronics Inc, Toronto, Canada), and a pressure sensor, to the preamplifier (PCU-2000; Millar Instruments). The ICP signal was acquired by using the MATLAB software, while the analysis protocol was programmed by W.J. The CRD protocol consisted of two $60 \mathrm{mmHg}$ CRD sessions in which the balloon was unfolded, immediately followed by 2 series of graded phasic distensions to constant pressures of 10, 20, 40, and $60 \mathrm{mmHg}$. Each CRD lasted 20 seconds and was applied at 4-minute interstimulus intervals. Data analysis was performed by using the method described in a previous study. ${ }^{27,28}$ The phasic component of the ICP (pICP) was extracted from the ICP signal recorded by applying the "DC Remove" process with a time constant of 1 second to exclude the slower tonic changes in ICP resulting from colonic smooth muscle activity and by applying the "root mean square amplitude" process with a time constant of 1 second to the resulting trace. The definition of visceromotor reflex (VMR) was the increase in the area under the curve of pICP during CRD over the mean values of the pre- and post-distension 20-second periods and VMR was calculated by using the MATLAB software (R2014b; The MathWorks, Natick, MA, USA). Each session of $\mathrm{CRD}$ was repeated twice, and the pre-, intra-, and post-CRD values were averaged for each pressure.

\section{Experimental Protocol}

The rats in the stress and no-stress groups were trained in Bollman cages for $60 \mathrm{~min} /$ day for 3 days before day 0 . All rats were exposed to 1 hour of WAS or sham WAS daily for 10 consecutive days (days 1-10). Body weight was measured every day before each WAS or sham WAS session, and FPO was monitored during each 1-hour WAS session for 10 days (days 1-10). On day 0 (baseline), day 10 (45-50 minutes after the end of the WAS session), and day 11 (24 hours after the last WAS session), the rats were subjected to the CRD protocol and VMR was measured in both groups. Immediately after the last $\mathrm{CRD}$ session on day 11, all rats were sacrificed by $\mathrm{CO}_{2}$ inhalation, and blood and distal colon samples were collected (Fig. 1).

\section{Immunohistochemistry}

The rats were sacrificed after the experiment. For histological analysis, a 1-cm length specimen of the distal colon was obtained from each rat and fixed using $10 \%$ buffered formalin. The paraffin embedded specimens were sectioned perpendicularly to the lumen

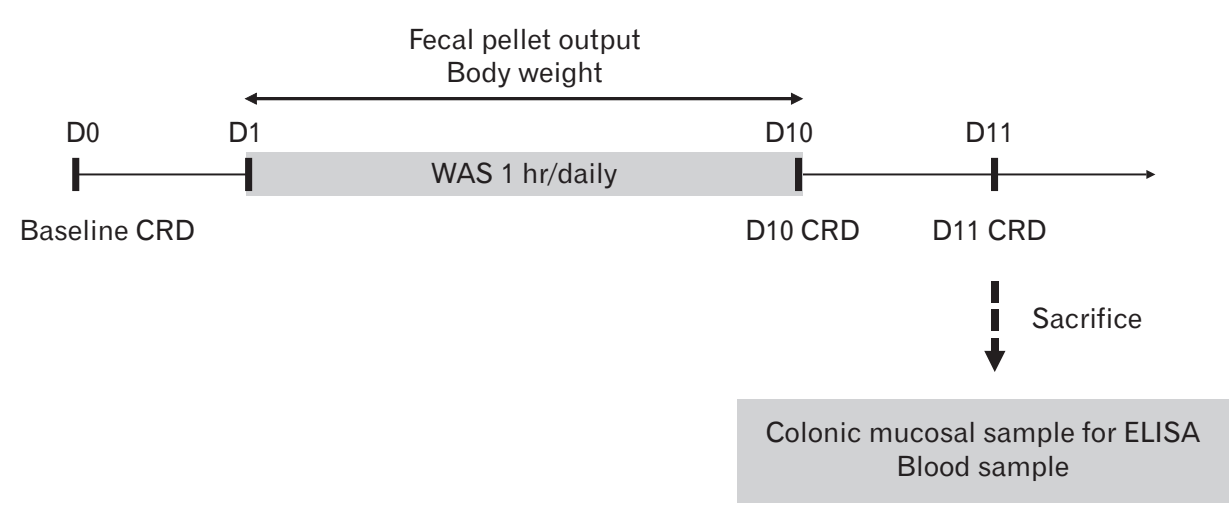

Figure 1. Experimental design. CRD, colorectal distention; WAS, water avoidance stress; D0, Day 0; D1, Day 1; D10, Day 10; D11, Day 11; ELISA, enzyme-linked immunosorbent assay. 
(section thickness, $4 \mu \mathrm{m}$ ), and mounted on a slide glass. The immunohistochemistry (IHC) stain was done by using an automatic immunostainer (BenchMark XT; Ventana Medical Systems, Tucson, AZ, USA) according to the manufacturer's instructions. The negative IHC control omitted the primary antibody. The slides were incubated with primary antibodies and a mouse monoclonal anti-mast cell tryptase antibody (Abcam Inc, Cambridge, MA, USA). Sections were then counterstained with hematoxylin for 4 minutes to stain the nucleus and then dehydrated, cleared, and mounted in synthetic mountant. Photographs of tryptase positive cells were obtained from 6 to 8 non-overlapping fields on 2 immunostained slides per rat under a light microscope (Carl Zeiss, Jena, Germany) linked to a computer-assisted image analysis system. The number of cells stained with the primary antibody was counted in all the photographs by the 3 researchers who were blinded to the animal groupings, and the cell numbers were expressed as visible mast cells in each high-power field (number of cells/hpf).
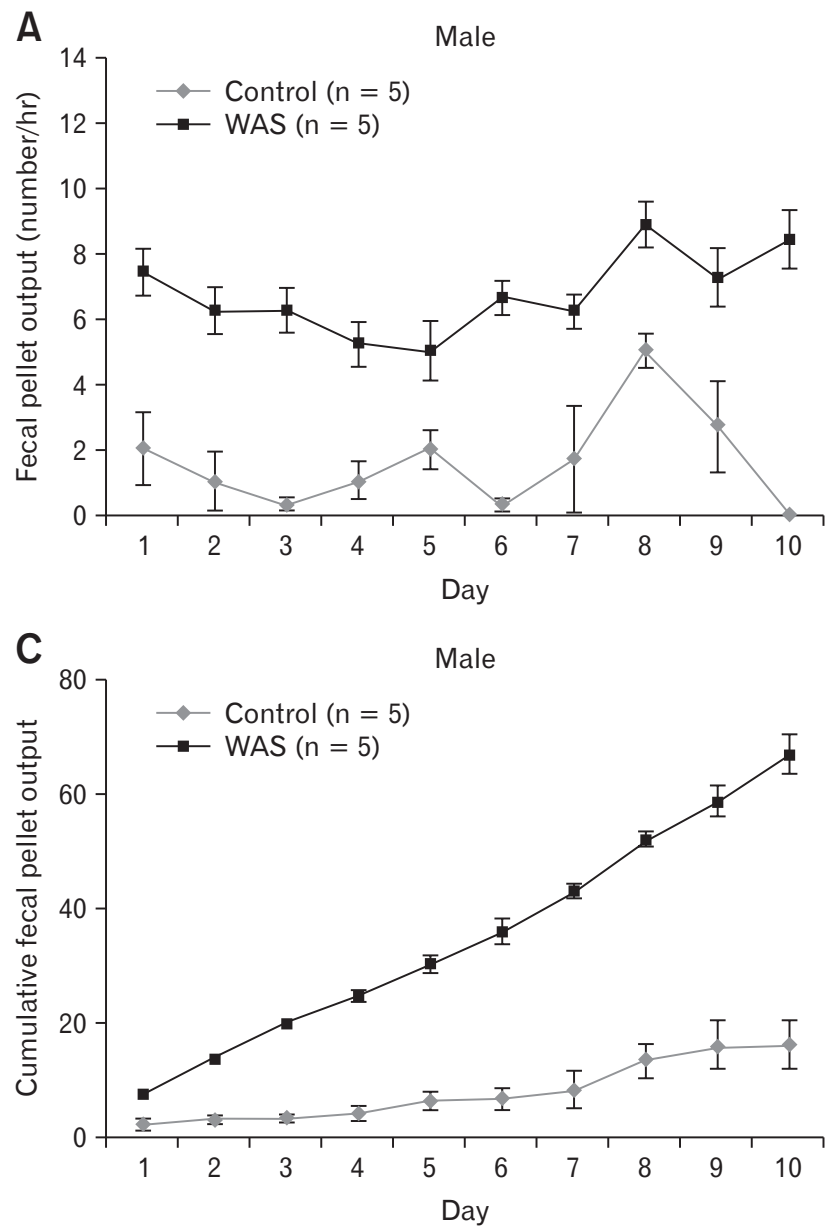

\section{Measurement of Mucosal Interleukin-1 $\beta$, Tumor Necrosis Factor- $\alpha$, and Interferon- $\gamma$ Levels}

Ten milligrams of mucosa were scraped and homogenized for 30 seconds with a Polytron homogenizer in $200 \mu \mathrm{L}$ of ice-cold lysis buffer $(200 \mathrm{mM} \mathrm{NaCl}, 5 \mathrm{mM}$ ethylenediaminetetraacetic acid, 10 $\mathrm{mM}$ Tris [pH 7.4], $10 \%$ glycerin, $1 \mathrm{mM}$ phenylmethanesulfonyl fluoride, leupeptin $1 \mu \mathrm{g} / \mathrm{mL}$, and aprotinin $28 \mu \mathrm{g} / \mathrm{mL}$ ). The cell suspensions were centrifuged at $13000 \mathrm{rpm}$ for 15 minutes. For IL-1 $\beta$, tumor necrosis factor- $\alpha(\mathrm{TNF}-\alpha)$ and IFN- $\gamma$ levels, kits from the R\&D Systems (Minneapolis, MN, USA) were used according to the manufacturer's protocols. Protein concentrations were measured using a Bio-Rad Protein Assay Kit (Bio-Rad Laboratories, Hercules, CA, USA). The level of each cytokine is expressed in picograms per milligram of protein. All the assays were performed in triplicate.
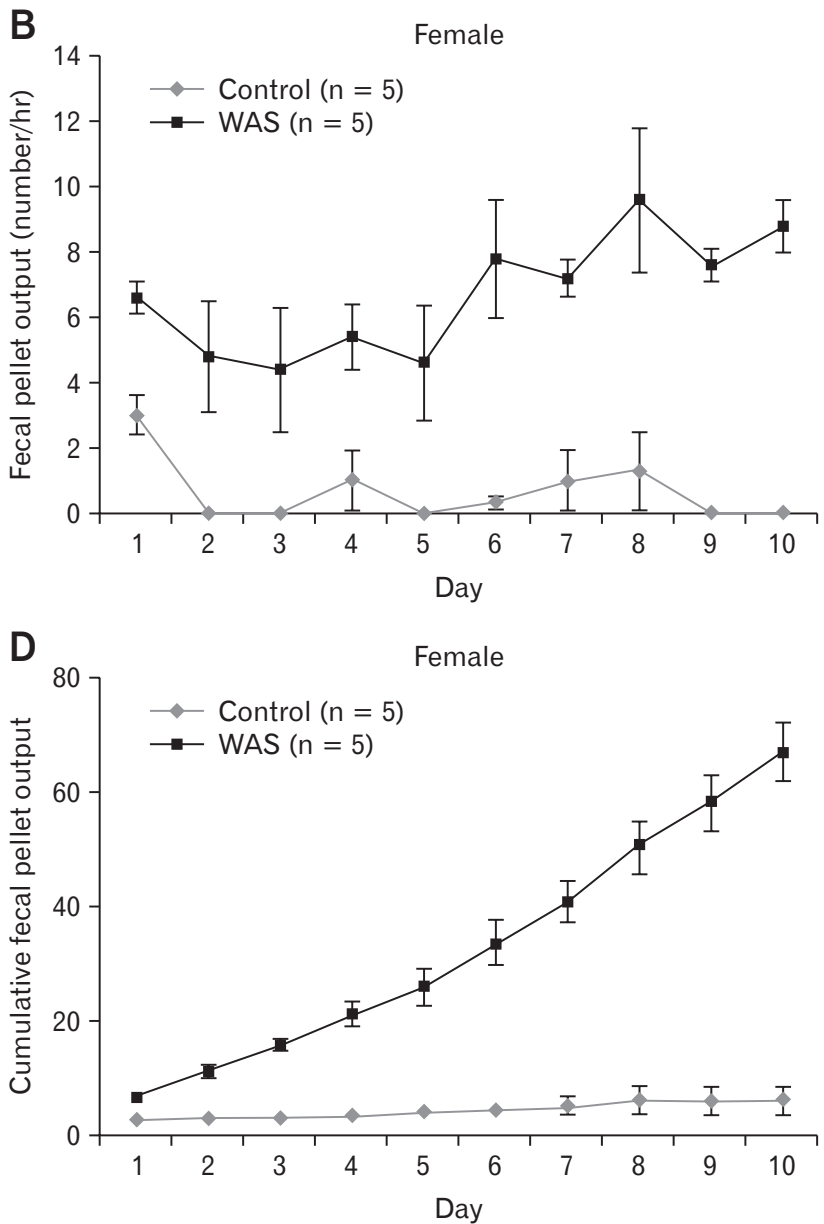

Figure 2. Fecal pellet output induced by repeated water avoidance stress (WAS). Data are expressed as means \pm SEM. 


\section{Statistical Methods}

Data are expressed as means \pm SEM. Sex-related differences in FPO and VMR to CRD over time between the WAS and nostress groups were analyzed by using one-way ANOVA or 2-way ANOVA followed by the Bonferroni post hoc test. Continuous and categorical variables were compared between the two groups (WAS and no-stress) by using the Mann-Whitney $U$ test and Fisher exact test, respectively. $P$-values of $<0.05$ were considered statistically significant. All statistical analyses were performed by using the SPSS version 20.0 software (SPSS Inc, Chicago, IL, USA).
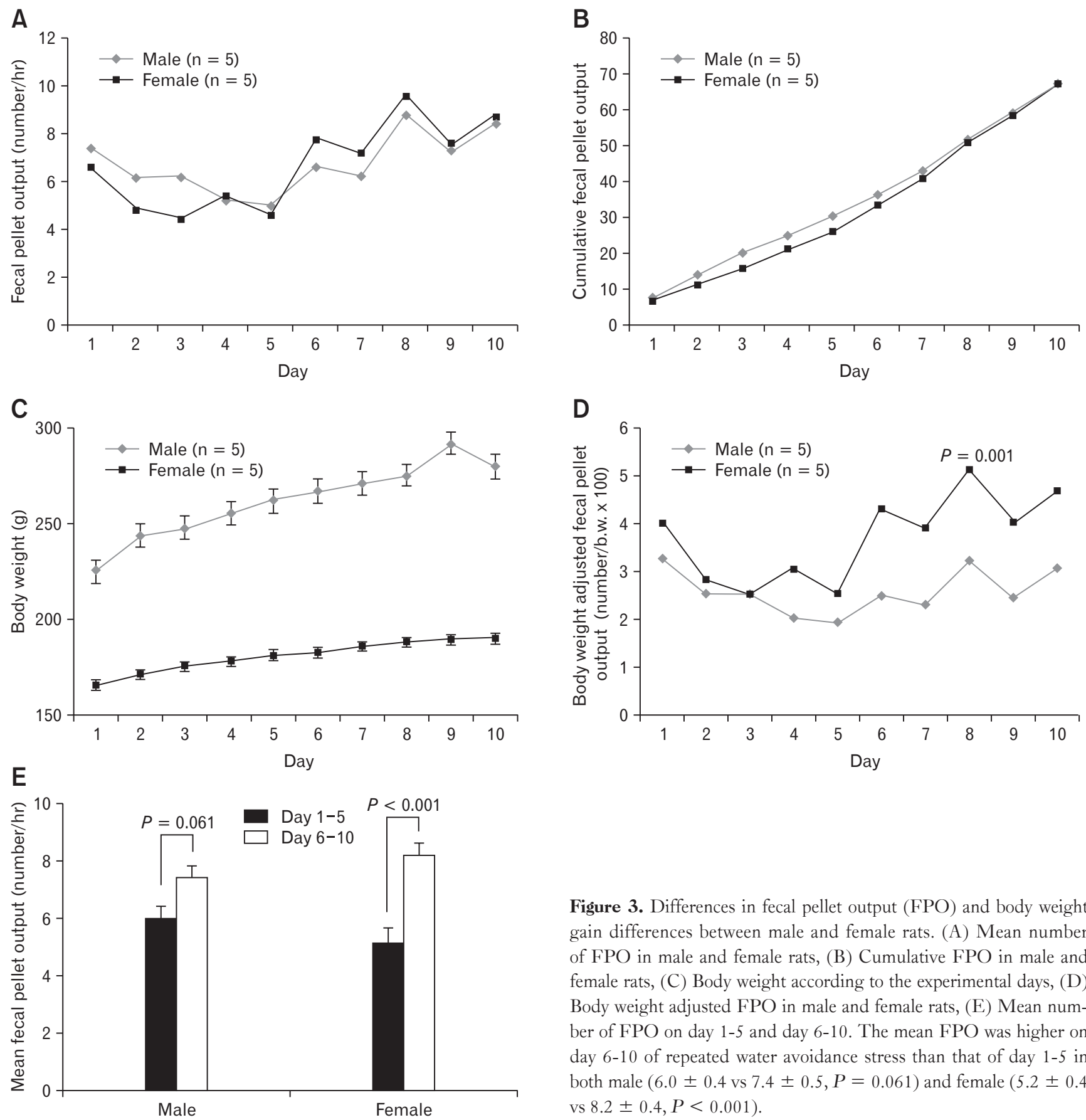

Figure 3. Differences in fecal pellet output (FPO) and body weight gain differences between male and female rats. (A) Mean number of FPO in male and female rats, (B) Cumulative FPO in male and female rats, (C) Body weight according to the experimental days, (D) Body weight adjusted FPO in male and female rats, (E) Mean number of FPO on day 1-5 and day 6-10. The mean FPO was higher on day 6-10 of repeated water avoidance stress than that of day $1-5$ in both male $(6.0 \pm 0.4$ vs $7.4 \pm 0.5, P=0.061)$ and female $(5.2 \pm 0.4$ vs $8.2 \pm 0.4, P<0.001)$. 


\section{Results}

\section{Sex-related Difference in Fecal Pellet Output and Body Weight Gain During Water Avoidance Stress}

The mean FPO in the WAS group increased significantly compared with that in the no-stress group during the experiment in both male $(6.7 \pm 0.1$ vs $1.6 \pm 0.2$ /hour; $P<0.05)$ and female rats (6.7 \pm 0.2 vs $0.7 \pm 0.1$ /hour; $P<0.05$; Fig. $2 \mathrm{~A}$ and $2 \mathrm{~B})$. The cumulative FPO also significantly differed between the WAS and no-stress groups in the later experimental period (Fig. 2C and 2D). The mean FPO (6.7 \pm 0.1 vs $6.7 \pm 0.2$ /hour $)$ and cumulative
FPO did not differ significantly between the male and female rats (Fig. 3A and 3B). Male rats had a significant higher body weight than female rats at the beginning of the experiment. Both male and female rats that were exposed to rWAS showed a consistent increase in body weight gain over time (on WAS day 10: $24.4 \pm 3.5 \%, \mathrm{n}=$ 5 vs $14.6 \pm 1.1 \%, \mathrm{n}=5$, respectively), which significantly differed between male and female rats $(P<0.05$; Fig. 3C). After adjustment for body weight, female rats had a significantly higher mean FPO than male rats (number/body weight $\times 100 ; P=0.001$; Fig. 3D). The mean FPO was higher in the last 5 days of rWAS (days 6-10) than in the first 5 days (days 1-5) in both male (6.0 \pm 0.4 vs $7.4 \pm 0.5, P=0.061)$ and female rats $(5.2 \pm 0.4$ vs $8.2 \pm 0.4, P$ $<0.001$; Fig. 3E).

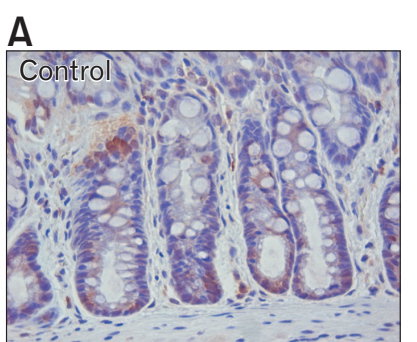

B

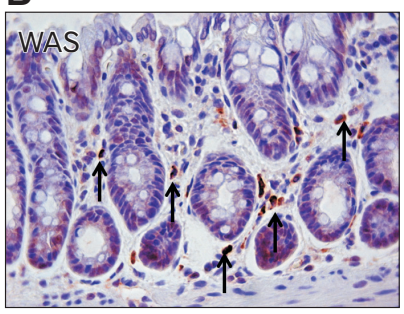

\section{C}

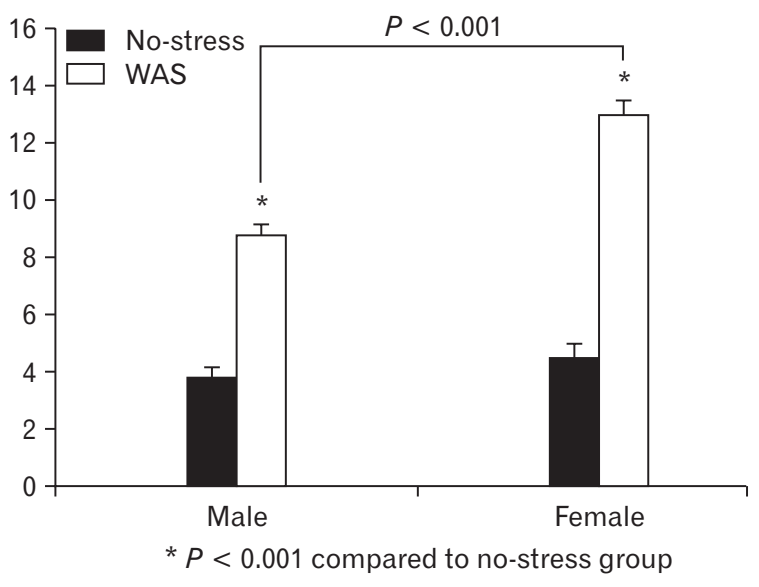

Figure 4. Number of mucosal mast cells detected after anti-mast cell tryptase antibody staining. Mucosal mast cell counts in the distal colon were higher in the water avoidance stress (WAS) group than in the no-stress group $(\mathrm{A}, \mathrm{B})$ in both male and female rats, and showed statistical significance in female rats $(8.8$ \pm 0.6 vs $13.0 \pm 0.9, P<0.001 ; \mathrm{C})$.
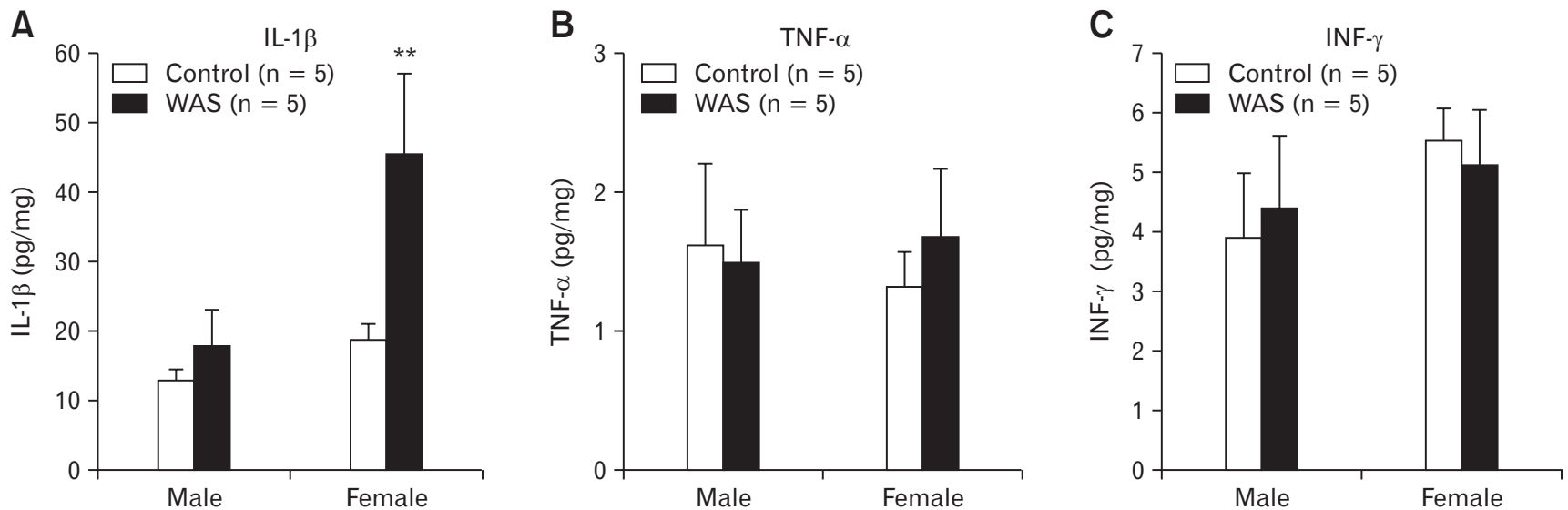

Figure 5. Colonic mucosal cytokine levels. (A) Mucosal interleukin (IL)-1 $\beta$ levels in the distal colon were significantly higher in female rats of the water avoidance stress (WAS) group than in those of the no-stress group. (B, C) No statistically differences in mucosal tumor necrosis factor (TNF)- $\alpha$ and interferon (INF)- $\gamma$ levels in the distal colon between male and female rats. ${ }^{*} P<0.05$. 


\section{Sex-related Difference in Mucosal Mast Cell Count in the Distal Colon}

Mucosal mast cell counts in the distal colon were significantly higher in the WAS group than in the no-stress group in both male $(8.8 \pm 0.6$ vs $3.8 \pm 0.3 ; P<0.001)$ and female rats $(13.0 \pm 0.9$ vs $4.5 \pm 0.5 ; P<0.001)$. The mucosal mast cell counts in the distal colon of the female rats of the WAS group were significantly higher than those of the male rats of the WAS group (13.0 \pm 0.9 vs $8.8 \pm$ $0.6 ; P<0.001$ ), showing a sex-specific difference (Fig. 4).

\section{Mucosal Cytokine Expression in the Distal Colon}

Mucosal IL-1 $\beta$ levels $(\mathrm{pg} / \mathrm{mL})$ in the distal colon were higher in the male rats of the WAS group than in the those of the no-stress group $(12.7 \pm 1.6$ vs $17.6 \pm 5.4, P>0.05)$, and significantly higher in female rats of the WAS group than in those of the nostress group (18.6 \pm 2.4 vs $45.3 \pm 11.7 ; P<0.05$; Fig. 5 A). The mucosal TNF- $\alpha$ level $(\mathrm{pg} / \mathrm{mL})$ in the distal colon was $1.6 \pm 0.6$ in male rats of the no-stress group, $1.5 \pm 0.4$ in male rats of the WAS group, $1.3 \pm 0.3$ in female rats of the no-stress group, and $1.7 \pm$ 0.5 in female rats of the WAS group (Fig. 5B). The mucosa INF- $\gamma$ level $(\mathrm{pg} / \mathrm{mL})$ in distal colon was $3.9 \pm 1.1$ in male rats of the nostress group, $4.4 \pm 1.2$ in male rats of the WAS group, $5.6 \pm 0.5$ in female rats of the no-stress group, and $5.1 \pm 0.9$ in female rats of the WAS group (Fig. 5C). No statistically significant difference in mean mucosal TNF- $\alpha$ and INF- $\gamma$ levels in distal colon were found
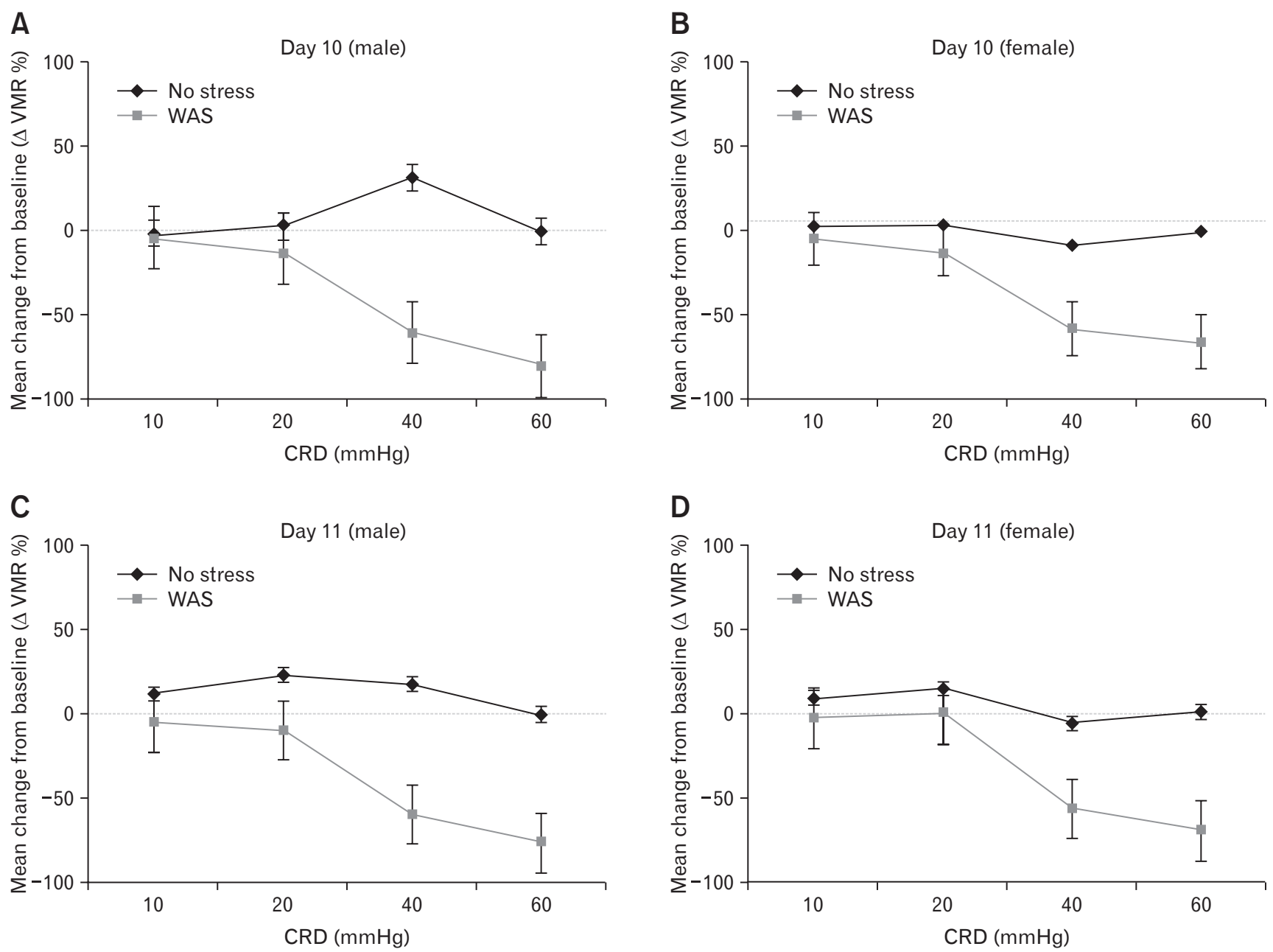

Figure 6. Visceral analgesic responses induced by repeated water avoidance stress (WAS) in male and female Wistar rats. (A, B) On day 10 of repeated WAS, decreased visceromotor response (VMR) to colorectal distension (CRD) (visceral analgesia) compared to baseline was observed at both 40 and $60 \mathrm{mmHg}$ in male rats and female rats. (C, D) On day 11, the same decreasing VMR pattern compared to baseline was observed at both 40 and $60 \mathrm{mmHg}$ in male rats and female rats. 
between male and female rats (Fig. 5B and 5C).

\section{Sex-related Difference in Visceromotor Response to Colorectal Distension}

In male and female rats of the no-stress group, no statistically significant changes in VMR to any CRD pressure were observed from baseline (day 0 ) to days 10 and 11 . On day 10 of rWAS, decreased VMR to CRD (visceral analgesia) compared with baseline was observed at both 40 and $60 \mathrm{mmHg}$ in male rats (VMR: $60.7 \%$ $\pm 18.7 \%$ and $80.4 \% \pm 19.2 \%$, respectively; $P<0.05)$ and female rats (VMR: $58.1 \% \pm 14.3 \%$ and $66.1 \% \pm 14.5 \%$, respectively; $P<0.05$; Fig. 6A and 6B). On day 11, the same decreasing VMR pattern compared with baseline was observed at both 40 and $60 \mathrm{mmHg}$ in male rats (VMR: $58.9 \% \pm 18.6 \%, 76.1 \% \pm$ $19.4 \%$, respectively; $P<0.05)$ and female rats (VMR: $57.4 \% \pm$ $12.6 \%, 70.1 \% \pm 13.4 \%$, respectively; $P<0.05$; Fig. $6 \mathrm{C}$ and $6 \mathrm{D}$ ). However, no statistically significant difference was observed in the decreased VMR to CRD between days 10 and 11, nor was a sexbased difference observed in the analgesic response to rWAS.

\section{Discussion}

Here, we demonstrated that chronic psychological stress increased colonic movement, especially in female rats, and that the distal colonic mucosal IL-1 $\beta$ level was significantly higher in female rats of the WAS group. Stress-induced visceral analgesia was observed in both sexes by using a noninvasive monometric catheter.

The selection of appropriate rat strains was important because each showed different anxiety traits. Wistar rat has been known to display high anxiety levels, and Gunter et $\mathrm{al}^{29}$ found that highly anxious Wistar-Kyoto rats exhibited an exaggerated VMR to CRD as compared with low/moderate-anxiety Fisher-344 (F344) and Sprague-Dawley (SD) rats. In our preliminary experiment, we also observed highly anxious behavior in Wistar rats subjected to WAS. Wistar rats showed higher FPO than did SD and F344 rats, especially female rats (data not shown). Anxiety is linked with visceral hypersensitivity, and rats, such as Wistar rats, being predisposed to heightened anxiety levels displayed dominant hypersensitivity, can be a suitable strain for stress-induced studies.

In our data, the body weight of both male and female rats markedly increased and the mean FPO was higher in the last 5 days of rWAS (days 6-10) than in the first 5 days (days 1-5) in both sexes, especially in female rats. We suggest that adaptation to a homotypic stressor occurred and the impact of stress may be reduced, thereby decreasing the inhibition of food intake. This can be reflected in the higher body weight gain during the last 5 days. Moreover, defecation is highly correlated with the content of feces, which is linked with the increase in food intake. Our data indicate that the female rats were more vulnerable to WAS, especially in the last 5 days of stress. This finding is similar to that of the previous study that demonstrated the sex-related differences after WAS, and showed that female rats had higher FPO than male rats. ${ }^{27}$ However, another study demonstrated the adaptation of a homotypic stressor in male rats that showed decreased FPO after 10 days of rWAS, ${ }^{8}$ which is rather different from our results. Currently, we cannot explain this difference, but some confounding stressors such as no enrichment, small cages, or cabinet-form housing might have affected the experiment results. Many human studies also demonstrated that women are more vulnerable to life stress, anxiety, and present exacerbated IBS symptoms under stress. ${ }^{20}$ The reason of the sex-related differences in IBS remains unknown. However, a previous study suggested that the relationship between ovarian and stress hormones may be important in the female predominance of IBS. $^{30}$

Our data showed female predominance in mast cell count and mucosal IL-1 $\beta$ levels. Mast cells and low-grade inflammation play important roles in some patients with IBS. ${ }^{14,16-18}$ The degranulation of intestinal mast cells can result in the release of histamine, serotonin, and cytokines. All these mediators are known to facilitate the response of afferent fibers that induce a varietyof visceral pain, which is the main symptom reported by patients with IBS. ${ }^{31}$ Previous studies have revealed that a mast cell stabilizer suppressed the stress-induced rectal hyperalgesia, proposing that it depends on mast cell degranulation. ${ }^{32}$ Bradesi et al ${ }^{8}$ reported that male Wistar rats subjected to rWAS showed anxiety-like behaviors, enhanced FPO, and increased mast cell counts and IL- $1 \beta$ and IFN- $\gamma$ expression levels. Santos et $\mathrm{al}^{33}$ examined the participation of mast cells in chronic stress response. After subjecting mast cell-deficient Ws/Ws rats and $+/+$ control rats to WAS or sham stress, they demonstrated that mucosal mast cell counts were increased in $+/+$ rats but not in Ws/Ws rats. Mast cell number and the amount of histamine secretion were higher in female rats than in the male rats. ${ }^{34}$ In addition, in human studies, Sohn et $\mathrm{al}^{35}$ showed that women with IBS had more mast cells than healthy women volunteers, and this trend was more predominant in women than in men. Barbara et $\mathrm{al}^{14}$ also showed significantly higher mast cell counts in female IBS patients than in male IBS patients. Mast cells are known to have high affinity to estrogen receptors, and estradiol has been shown to augment mast cell secretion. ${ }^{30}$ These previous results suggested sex-related differences in IBS, taking into account the immune responses. 
A recently validated noninvasive method that uses manometry showed that rWAS can induce stress-related visceral analgesia in response to $\mathrm{CRD}{ }^{27,28}$ This method has an advantage over the electromyographic method in that it does not require surgery and postsurgical antibiotics and analgesics, which can affect visceral pain responses. ${ }^{9}$ Dual visceral pain responses (hyperalgesia vs analgesia) have been reported in rodents exposed to psychological stressors, and factors that can affect the response to psychological stressors include the tested animal, the social environment of the animal, handling of the investigator, and the methods used to record VMR. ${ }^{19}$ Therefore, these factors should be carefully taken into account in the design, and interpretation of results of animal studies. Although we found increased colonic movement and significant sex-related differences in mucosal mast cell count and higher colonic mucosal IL-1 $\beta$ levels in rWAS-female rats, we did not observe sex-related differences in stress-induced visceral analgesia. In the previous study, rWAS for 4 days induced a strong visceral analgesia at 40 $\mathrm{mmHg}$ in both sexes. However, 24 hours later (day 5), female rats exhibited visceral hyperalgesia at $60 \mathrm{mmHg}$ and the VMR of male rats returned to normal. ${ }^{27}$ In contrast to the previous study, the present study showed sustained visceral analgesia on day 11 in both sexes. Our study applied longer rWAS (for 10 days) and both sexes of rats showed higher FPO in the last 5 days of rWAS, which is inconsistent with the findings of the previous study. The difference in stress duration seemed to induce different VMR results. Hence, further studies are needed.

In the field of somatic pain, opioid- or non-opioid-dependent inhibitory systems can mediate the mechanisms of stress-induced analgesia. ${ }^{36}$ Briefly, stress lead to the release of endogenous opioids from presynaptic neurons and endogenous cannabinoids from postsynaptic neurons. The activation of cannabinoids or opioid $\left(\mu-, \kappa^{-}\right.$, and $\sigma$-) receptors on $\gamma$-aminobutyric acid (GABA) ergic interneurons inhibits the release of GABA and facilitates neurotransmission at glutamatergic synapses, resulting in activation of the descending inhibitory pain pathway. ${ }^{36}$ A non-opioid, neurotensindependent visceral analgesia was observed in SD rats after exposure to acute WAS, with male rats exhibiting stronger analgesia than female rats. ${ }^{37}$ In another study, psychological stress induced an immediate post-stress visceral analgesia mediated by an opiate signal in females and a naloxone-independent mechanism in males, as well as hyperalgesia at 24 hours after repeated stress only in females. ${ }^{27}$ These data highlight the importance of sex-specific interventions to modulate visceral pain responses to stress. ${ }^{27}$ In the field of visceral responses, most of the preclinical studies conducted were in male rodents, ${ }^{37}$ but few studies in females indicated that sex hormones may have an effect on visceral sensitivity in rodents. ${ }^{38,39}$ Some studies did not even mention the sex of experimental rodents. ${ }^{33}$ The use of a balanced proportion of male and female rodents in preclinical studies and application of sex-based analysis are needed to elucidate sex-related differences in the pathophysiological mechanism of IBS. In addition, as the response of new drug for IBS and constriction is known to be different depending on sex ${ }^{40-43}$ this approach will be more important and useful.

The limitations of this experiment are as follows: we did not exactly measure the amount of food intake per day, which is reported to be linked with the frequency and amount of fecal pellets, and which can influence the body weight gain in the last 5 days of the experiment. The food intake during the 10-day period should have been taken into account to clarify the relationship among body weight, amount of feces, and stress response. In addition, we used a relatively small animal cage without enrichment and the rats were housed in a cage that was kept in a cabinet. We think these experimental environments might have acted as a confounding factor for stress responses.

In conclusion, chronic stress increased bowel frequency, mucosal mast cell count, and mucosal IL-1 $\beta$ levels, especially in female Wistar rats. Chronic stress also induced visceral analgesia with the noninvasive manometric method in rats of both sexes. However, the stress-induced visceral analgesia after 10 days of WAS did not differ significantly between the sexes. Hence, the underlying mechanism needs to be further evaluated.

Acknowledgements: The authors are grateful to Prof. Yvette Taché and Muriel Larauche of the UCLA for the opportunity given to Nayoung Kim to observe the experiment visceromotor response to colorectal distension with repeated water avoidance stress in 2014. Currently, this noninvasive manomteric technique is not available in Korea, and we highly appreciate their support. Ju Yup Lee and Ryoung Hee Nam learned this experimental process from Nayoung Kim and Yong Sung Kim. In addition, Sam Jundler, President of G\&J Electronics Inc, helped set up the barostat experiment in our institute. The analysis protocol for pressure signal was programed by Wonjun Jo by using the MATLAB software.

Financial support: This work was supported by grant (No. 062013-099) from the Seoul National University Bundang Hospital Research.

Conflicts of interest: None. 
Author contributions: Ju Yup Lee analyzed and interpreted the data and wrote a manuscript; Nayoung Kim designed, organized, and mediated the present study and supervised the manuscript; Yong Sung Kim supervised experimental design and the manuscript; Ryoung Hee Nam, Min Hee Ham, Youngkwang Shim, and Yoon Jin Choi performed the experiments; Hye Seung Lee performed the immunohistochemistry of mucosal mast cell; Wonjun Jo programmed analysis protocol of the intracolonic pressure signal which was acquired using MATLAB software; Hyuk Yoon and and Cheol Min Shin performed the statistical analysis; and Dong Ho Lee advised design and supervised preparing manuscript.

\section{References}

1. Longstreth GF, Thompson WG, Chey WD, Houghton LA, Mearin F, Spiller RC. Functional bowel disorders. Gastroenterology 2006;130:1480-1491.

2. Barbara $G$, Cremon $C$, Carini $G$, et al. The immune system in irritable bowel syndrome. J Neurogastroenterol Motil 2011;17:349-359.

3. Barbara G, De Giorgio R, Stanghellini V, Cremon C, Salvioli B, Corinaldesi R. New pathophysiological mechanisms in irritable bowel syndrome. Aliment Pharmacol Ther 2004;20(suppl 2):1-9.

4. Chang $\mathrm{L}$. The role of stress on physiologic responses and clinical symptoms in irritable bowel syndrome. Gastroenterology 2011;140:761-765.

5. Taché Y, Million M. Role of corticotropin-releasing factor signaling in stress-related alterations of colonic motility and hyperalgesia. J Neurogastroenterol Motil 2015;21:8-24.

6. Larauche M, Gourcerol G, Wang L, et al. Cortagine, a CRF1 agonist, induces stresslike alterations of colonic function and visceral hypersensitivity in rodents primarily through peripheral pathways. Am J Physiol Gastrointest Liver Physiol 2009;297:G215-G227.

7. Stengel A, Taché Y. Corticotropin-releasing factor signaling and visceral response to stress. Exp Biol Med (Maywood) 2010;235:1168-1178.

8. Bradesi S, Schwetz I, Ennes HS, et al. Repeated exposure to water avoidance stress in rats: a new model for sustained visceral hyperalgesia. Am J Physiol Gastrointest Liver Physiol 2005;289:G42-G53.

9. Larauche M, Mulak A, Taché Y. Stress-related alterations of visceral sensation: animal models for irritable bowel syndrome study. J Neurogastroenterol Motil 2011;17:213-234.

10. Mulak A, Larauche M, Taché Y. Psychological stress induces visceral analgesic or hyperalgesic response in rodents: a role of preconditions. Front Gastrointest Res 2012;30:106-114.

11. Larauche M, Gourcerol G, Million M, Adelson DW, Taché Y. Repeated psychological stress-induced alterations of visceral sensitivity and colonic motor functions in mice: influence of surgery and postoperative single housing on visceromotor responses. Stress 2010;13:343-354.

12. Ramsay DB, Stephen S, Borum M, Voltaggio L, Doman DB. Mast cells in gastrointestinal disease. Gastroenterol Hepatol (N Y) 2010;6:772-777.

13. O'Sullivan M, Clayton N, Breslin NP, et al. Increased mast cells in the irritable bowel syndrome. Neurogastroenterol Motil 2000;12:449-457.

14. Barbara G, Stanghellini V, De Giorgio R, et al. Activated mast cells in proximity to colonic nerves correlate with abdominal pain in irritable bowel syndrome. Gastroenterology 2004;126:693-702.

15. Söderholm JD, Yang PC, Ceponis P, et al. Chronic stress induces mast cell-dependent bacterial adherence and initiates mucosal inflammation in rat intestine. Gastroenterology 2002;123:1099-1108.

16. Chadwick VS, Chen W, Shu D, et al. Activation of the mucosal immune system in irritable bowel syndrome. Gastroenterology 2002;122:17781783.

17. Spiller R, Garsed K. Postinfectious irritable bowel syndrome. Gastroenterology 2009;136:1979-1988.

18. Chang L, Adeyemo M, Karagiannides I, et al. Serum and colonic mucosal immune markers in irritable bowel syndrome. Am J Gastroenterol 2012;107:262-272.

19. Lovell RM, Ford AC. Global prevalence of and risk factors for irritable bowel syndrome: a meta-analysis. Clin Gastroenterol Hepatol 2012;10:712-721, e4.

20. Meleine M, Matricon J. Gender-related differences in irritable bowel syndrome: potential mechanisms of sex hormones. World J Gastroenterol 2014;20:6725-6743.

21. Mulak A, Taché Y, Larauche M. Sex hormones in the modulation of irritable bowel syndrome. World J Gastroenterol 2014;20:2433-2448.

22. Chaban V. Estrogen and visceral nociception at the level of primary sensory neurons. Pain Res Treat Published Online First: 1 Jan 2012. doi: 10.1155/2012/960780.

23. Ji Y, Bai G, Cao DY, Traub RJ. Estradiol modulates visceral hyperalgesia by increasing thoracolumbar spinal GluN2B subunit activity in female rats. Neurogastroenterol Motil 2015;27:775-786.

24. Sanoja R, Cervero F. Estrogen-dependent changes in visceral afferent sensitivity. Auton Neurosci 2010;153:84-89.

25. Galligan JJ, Patel BA, Schneider SP, et al. Visceral hypersensitivity in female but not in male serotonin transporter knockout rats. Neurogastroenterol Motil 2013;25:e373-e381.

26. Winston JH, Li Q, Sarna SK. Chronic prenatal stress epigenetically modifies spinal cord BDNF expression to induce sex-specific visceral hypersensitivity in offspring. Neurogastroenterol Motil 2014;26:715-730.

27. Larauche M, Mulak A, Kim YS, Labus J, Million M, Taché Y. Visceral analgesia induced by acute and repeated water avoidance stress in rats: sex difference in opioid involvement. Neurogastroenterol Motil 2012;24:1031-e547.

28. Larauche M, Mulak A, Yuan PQ, Kanauchi O, Taché Y. Stress-induced visceral analgesia assessed non-invasively in rats is enhanced by prebiotic diet. World J Gastroenterol 2012;18:225-236.

29. Gunter WD, Shepard JD, Foreman RD, Myers DA, Greenwood-Van Meerveld B. Evidence for visceral hypersensitivity in high-anxiety rats. Physiol Behav 2000;69:379-382.

30. Cremon C, Gargano L, Morselli-Labate AM, et al. Mucosal immune activation in irritable bowel syndrome: gender-dependence and association with digestive symptoms. Am J Gastroenterol 2009;104:392-400.

31. Eutamene H, Theodorou V, Fioramonti J, Bueno L. Acute stress modulates the histamine content of mast cells in the gastrointestinal tract 
through interleukin-1 and corticotropin-releasing factor release in rats. J Physiol 2003;553(Pt 3):959-966.

32. Gué M, Del Rio-Lacheze C, Eutamene H, Théodorou V, Fioramonti J, Buéno L. Stress-induced visceral hypersensitivity to rectal distension in rats: role of CRF and mast cells. Neurogastroenterol Motil 1997;9:271279.

33. Santos J, Yang PC, Soderholm JD, Benjamin M, Perdue MH. Role of mast cells in chronic stress induced colonic epithelial barrier dysfunction in the rat. Gut 2001;48:630-636.

34. Bradesi S, Eutamene H, Theodorou V, Fioramonti J, Bueno L. Effect of ovarian hormones on intestinal mast cell reactivity to substance P. Life Sci 2001;68:1047-1056.

35. Sohn W, Lee OY, Lee SP, et al. Mast cell number, substance $P$ and vasoactive intestinal peptide in irritable bowel syndrome with diarrhea. Scand J Gastroenterol 2014;49:43-51.

36. Butler RK, Finn DP. Stress-induced analgesia. Prog Neurobiol 2009;88:184-202.

37. Gui X, Carraway RE, Dobner PR. Endogenous neurotensin facilitates visceral nociception and is required for stress-induced antinociception in mice and rats. Neuroscience 2004;126:1023-1032.
38. Aloisi AM, Affaitati G, Ceccarelli I, et al. Estradiol and testosterone differently affect visceral pain-related behavioural responses in male and female rats. Eur J Pain 2010;14:602-607.

39. Ji Y, Tang B, Traub RJ. The visceromotor response to colorectal distention fluctuates with the estrous cycle in rats. Neuroscience 2008;154:15621567.

40. Eswaran S, Guentner A, Chey WD. Emerging pharmacologic therapies for constipation-predominant irritable bowel syndrome and chronic constipation. J Neurogastroenterol Motil 2014;20:141-151.

41. Lee HH, Choi YY, Choi MG. The efficacy of hypnotherapy in the treatment of irritable bowel syndrome: a systematic review and meta-analysis. J Neurogastroenterol Motil 2014;20:152-162.

42. Ke M, Tack J, Quigley EM, et al. Effect of prucalopride in the treatment of chronic constipation in Asian and non-Asian women: a pooled analysis of 4 randomized, placebo-controlled studies. J Neurogastroenterol Motil 2014;20:458-468.

43. Lee TH, Choi SC, Park MI, et al. Constipation misperception is associated with gender, marital status, treatment utilization and constipation symptoms experienced. J Neurogastroenterol Motil 2014;20:379-387. 